Background and aims Acute kidney injury (AKI) is a common consequence of perinatal asphyxia (PA). Therapeutic hypothermia $(\mathrm{TH})$ used for neuroprotection in hypoxic-ischaemic-encephalopathy (HIE) may also ameliorate kidney injury. AKI can be associated with more severe PA, but association with worse neurological outcome remains to be proven. We aim to evaluate the incidence of AKI in neonates under $\mathrm{TH}$ and the association with HIE grades and outcome.

Methods A total of 51 cooled infants were reviewed using a prospectively collected database. Modified AKIN criteria were used to classify AKI. We studied perinatal, resuscitation and clinical data during admission to the NICU. Outcome of interest was duration of NICU stay, predictable neurologic outcome and mortality.

Results AKI was found in 17 of 51 neonates (33\%). Based on the modified AKIN criteria, 13, 1, and 3 patients had stages I, II, and III, respectively. AKI was more frequent in HIE $2 / 3$ than in the rare HIE 1 infants that were cooled $(\mathrm{p}<0.03)$ and in those with worse base deficit at birth $(p<0.05)$. A significant association was found between vancomycin and gentamicin use and AKI $(\mathrm{p}<0.01)$. Renal replacement therapy was needed in $2 / 3$ of AKIN stage III infants. NICU stay, mortality and predicted adverse neurologic outcomes were not associated with AKI.

Conclusions AKI was less frequent in our cohort than the one previously described in non-cooled newborns. More severely asphyxiated neonates were more likely to experience AKI, but AKI was not related to worse outcomes.

\section{PO-0395 SCAVENGING OF EXTRACELLULAR HAEMOGLOBIN MODIFIES THE MONOCYTE-MACROPHAGE RECRUITMENT AND DIFFERENTIATION IN THE INTRAVENTRICULAR SPACE FOLLOWING IVH}

${ }^{1} \mathrm{M}$ Gram, ${ }^{1} \mathrm{~S}$ Sveinsdottir, ${ }^{1} \mathrm{~S}$ Vallius, ${ }^{1} \mathrm{~K}$ Sveinsdottir, ${ }^{2} \mathrm{M}$ Cinthio, ${ }^{1} \mathrm{D}$ Ley. ${ }^{1}$ Pediatrics, Lund University, Lund, Sweden; ${ }^{2}$ Electrical Measurments, Lund University, Lund, Sweden

10.1136/archdischild-2014-307384.1041

Introduction Severe cerebral intraventricular haemorrhage (IVH) in preterm infants continues to be a major clinical problem. To date, no available therapy prevents infants from neurologic sequel following IVH. Recruitment of monocytes-macrophages and periventricular infiltration is a key step in the inflammatory response leading to brain damage. The sequence of the recruitment and profiling of monocytes-macrophages following IVH is not well characterised. We have previously shown that extracellular haemoglobin induces chemotactic cytokines following IVH. Haptoglobin is a haemoglobin scavenger and could potentially protect the immature brain from the detrimental effects of haemoglobin.

Objective To characterise the recruitment and differentiation of monocytes-macrophages in the intraventricular space following IVH and to investigate if haemoglobin scavenging with haptoglobin alters the recruitment and differentiation.

Methods Using a preterm rabbit pup model of IVH we characterised the immune cell recruitment and differentiation in intraventricular cerebrospinal fluid (CSF) at 24 to $72 \mathrm{~h}$ following haemorrhage. Using flow cytometry, immunohistochemistry and mRNA and protein analysis we characterised the systemic and CSF infiltrating macrophages in animals with IVH, sham controls and animals treated with intraventricular injections of haptoglobin.

Results Following IVH, there is an infiltration of M1 macrophages into the intraventricular CSF. Intraventricular introduction of the haemoglobin-scavenger haptoglobin modifies them into alternative M2 macrophages, expressing CD163. This causes a subsequent in vivo clearance of the accumulated haemoglobin.

Conclusion Following IVH, intraventricular haptoglobin treatment modifies macrophage differentiation, initiating clearance of extracellular haemoglobin. Treatment of haptoglobin might be a feasible approach to protect the immature brain following IVH.

\section{P0-0396 THE SIGMA-1 RECEPTOR AGONIST PRE-084 PROTECTS AGAINST GLUTAMATE INDUCED NEUROTOXICITY IN PRIMARY HIPPOCAMPAL NEURONS}

${ }^{1}$ E Griesmaier, ${ }^{1} \mathrm{M}$ Urbanek, ${ }^{1} \mathrm{~K}$ Stock, ${ }^{2} \mathrm{M}$ Hermann, ${ }^{1} \mathrm{~A}$ Posod, ${ }^{3} \mathrm{RI}$ Stanika, ${ }^{3} \mathrm{GJ}$ Obermair, ${ }^{1} \mathrm{U}$ Kiechl-Kohlendorfer. 'Paediatrics II Neonatology, Innsbruck Medical University, Innsbruck, Austria; ${ }^{2}$ Anaesthesiology and Critical Care Medicine, Innsbruck Medical University, Innsbruck, Austria; ${ }^{3}$ Physiology and Medical Physics, Innsbruck Medical University, Innsbruck, Austria

\subsection{6/archdischild-2014-307384.1042}

Background Prematurity is a major determinant of neonatal mortality and morbidity. The number of preterm birth is still on the rise. Recently we and others could demonstrate neuroprotective effects of sigma-1 receptor ligands in adult and newborn animal models of brain injury. Since sigma-1 receptor agonists are already undergoing clinical trials in adult neurological disease, they might be considered a promising therapeutic option also in preterm brain injury. We have previously shown that the selective sigma-1 receptor agonist PRE-084 (2-(4-morpholinethyl)1-phenylcyclohexane-carboxylate) protects against neonatal excitotoxic brain injury in vivo. The aim of the present study was to investigate whether PRE-084 is able to prevent neurotoxicity following glutamate exposure in vitro.

Methods Cultured primary hippocampal neurons (day in vitro 10) were pre-treated with PRE-084 before glutamate was applied. Subsequently cell death was quantified by means of PI/ calcein - AM staining using live confocal microscopy. Neurons were randomly assigned to one of the following groups: i) control, ii) glutamate or iii) glutamate+PRE-084. PRE-084 was applied in two dosages $(10$ and $100 \mu \mathrm{M})$ prior to glutamate.

Results The application of PRE-084 significantly reduced the percentage of dead cells (PRE-084 $10 \mu \mathrm{M}$ : 22.09 (20.50;28.84) and $100 \mu \mathrm{M}$ : $25.87(18.77 ; 33.40))$ compared to the untreated glutamate control group $43.56(39,86 ; 46.02)$.

Conclusion Our data show that administration of PRE-084 protects against glutamate induced cell death in primary hippocampal neurons. PRE-084 shows considerable promise as a therapeutic strategy in preterm brain injury and might provide an adequate means of combating this major cause of neurological disability in infancy.

\section{P0-0397 THE NEUROPEPTIDE SECRETONEURIN IS PROTECTIVE IN ESTABLISHED IN VIVO AND IN VITRO MODELS OF NEONATAL BRAIN INJURY}

${ }^{1} \mathrm{E}$ Griesmaier, ${ }^{1} \mathrm{~K}$ Wechselberger, ${ }^{1} \mathrm{~A}$ Posod, ${ }^{1} \mathrm{~K}$ Stock, ${ }^{2} \mathrm{RI}$ Stanika, ${ }^{2} \mathrm{GJ}$ Obermair, ${ }^{3} \mathrm{M}$ Hermann, ${ }^{1} \mathrm{U}$ Kiechl-Kohlendorfer, ${ }^{1} \mathrm{M}$ Urbanek. ${ }^{1}$ Department of Paediatrics II Neonatology, Innsbruck Medical University, Innsbruck, Austria; ${ }^{2}$ Department of Physiology and Medical Physics, Innsbruck Medical University, Innsbruck, Austria; ${ }^{3}$ Department of Anaesthesiology and Critical Care Medicine, Innsbruck Medical University, Innsbruck, Austria

10.1136/archdischild-2014-307384.1043 
Background Hypoxic-ischaemic encephalopathy leads to neurologic impairment or even death. Secretoneurin (SN), a neuropeptide with angiogenic and anti-apoptotic properties, provided strong neuroprotection in an adult animal model of cerebral ischaemia.

Aim To evaluate the effect of $\mathrm{SN}$ in established in vivo and in vitro models of neonatal hypoxic-ischaemic brain injury.

Methods Seven day old mice underwent unilateral common carotid artery ligation, followed by exposure to hypoxia (8\% oxygen). Thereafter, mouse pups were randomly injected intraperitoneally with $\mathrm{SN}(0.25 \mu \mathrm{g} / \mathrm{g}$ body weight $)$ or vehicle. As endpoint we determined the histological injury score and the number of caspase- 3 positive cells $24 \mathrm{~h}$ after the insult. Primary cultured hippocampal neurons were treated with oxygen glucose deprivation (OGD) on day 10. Neurons were assigned to the following groups: i) control ii) OGD iii) $\mathrm{OGD}+\mathrm{SN}(1,10$ or 50 $\mu \mathrm{g} / \mathrm{l})$. As primary outcome parameter, cell death was evaluated via real time live confocal imaging using calcein-AM and propidium iodide (PI).

Results SN displayed a non-significant trend to lower mean values of histological injury score compared to control $(n=11-12$, $\mathrm{p}>0.05)$ and significantly reduced the number of cells stained positively for activated caspase-3 $(\mathrm{n}=6, \mathrm{p}<0.05)$. In vitro $\mathrm{SN}$ application on hippocampal neurons $(\mathrm{OGD}+\mathrm{SN})$ significantly reduced the number of dead cells assessed by the $\mathrm{PI} /$ calcein ratio compared with the untreated OGD group $(\mathrm{n}=8, \mathrm{p}<0.05)$.

Conclusion We provide first evidence that $\mathrm{SN}$ is neuroprotective in established in vitro and in vivo models of neonatal hypoxicischaemic brain injury and might therefore be considered a promising therapeutic option.

\section{PO-0398 INVOLVEMENT OF THE CORPUS CALLOSUM AFTER PERINATAL ASPHYXIA DEMONSTRATED USING DIFFUSION WEIGHTED MRI IS RELATED TO NEURODEVELOPMENTAL OUTCOME}

T Alderliesten, Y Khalil, C Koopman-Esseboom, MJNL Benders, IC van Haastert, LS de Vries, F Groenendaal. Department of Neonatology, Wilhelmina Children's Hospital University Medical Center Utrecht, Utrecht, Netherlands

\subsection{6/archdischild-2014-307384.1044}

Background Previous studies in 10-year-old children have shown an association between size of the corpus callosum and neurodevelopmental outcome.

Aim In the present study we examined whether diffusion changes in the corpus callosum shortly after birth are associated with outcome in full-term infants with perinatal asphyxia.

Subjects/methods From 2002 to 2013, 127 full-term infants with perinatal asphyxia were examined using diffusion weighted MRI within 7 days after birth (median 4 days; IQR 2 days). To calculate ADC values, regions of interest were manually selected in the anterior and posterior part of the corpus callosum, and ADC values were related to outcome. Adverse outcome was defined as death $(\mathrm{n}=33)$, cerebral palsy or delayed

\begin{tabular}{llll} 
Abstract PO-0398 Table 1 & \\
\hline & Favourable & CP or delayed development & Died \\
Corpus callosum anterior & $1094 \pm 131$ & $1033 \pm 286$ & $899 \pm 233$ \\
Corpus callosum posterior & $1064 \pm 130$ & $921 \pm 265$ & $812 \pm 225$ \\
\hline
\end{tabular}

development at 18 moths (Griffiths DQ <85): $n=12$. Sixtynine of the infants $(54.3 \%)$ received therapeutic hypothermia. Results ADC values $\left(10^{-6} \mathrm{~mm}^{2} / \mathrm{s}\right)$ showed anterior-to-posterior differences, and values were analysed separately. ADC values are presented in the table.

Differences between groups were significant (ANOVA $\mathrm{p}<$ 0.001).

Conclusions Low ADC values during the first 7 days after birth, in particular of the posterior part of the corpus callosum, are associated with an adverse outcome in asphyxiated term neonates.

\section{PO-0399 NEURODEVELOPMENTAL AND BEHAVIOUR OUTCOME OF PRETERMS (GA}

${ }^{1} \mathrm{~K}$ Gücüyener, ${ }^{1} \mathrm{~S}$ Soysal, ${ }^{2} \mathrm{E}$ Ergenekon, ${ }^{3} \mathrm{~T}$ Bedir, ${ }^{2} \mathrm{E}$ Kazanci, ${ }^{2} \mathrm{H}$ Hirfanoglu, ${ }^{2} \mathrm{E}$ Koc, ${ }^{2} \mathrm{C}$ Turkyilmaz, ${ }^{2} \mathrm{E}$ Onal, ${ }^{2} \mathrm{Y}$ Atalay. ${ }^{1}$ Pediatric Neurology, Gazi University Faculty of Medicine, Ankara, Turkey; ${ }^{2}$ Neonatology, Gazi University Faculty of Medicine, Ankara, Turkey; ${ }^{3}$ Pediatrics, Gazi University Faculty of Medicine, Ankara, Turkey

\subsection{6/archdischild-2014-307384.1045}

Background and aims We aimed to identify the neurodevelopmental and behaviour outcome of preterms (GA $<30 \mathrm{wks}$ ) at 8 years by age appropriate psychometric evaluations to see whether tests used at younger ages could predict worst outcomes at older ages in relation to some neonatal factors.

Method Along with neurologic examinations, 33 infants were prospectively evaluated at 3, 6, 12,18, 24 months of corrected age with Bayley Scales of Infant Development - II (BSID-II), at 3, 5 years with Stanford-Binet, at 8 years with WISC-R.

Results $72 \%$ of children had no (IQ $>85$ ), $24.3 \%$ had mild (IQ 74-84), 3\% had major (IQ $<70$, blindness) impairments. $24.2 \%$ had special education, 15.2\% ADHD, 6.1\% autism, 9.1\% learning/language, $6.1 \%$ anxiety disorders. The probability of neurodevelopmental test and IQ scores of VLBW infants $<1000 \mathrm{gr}$ being lower than healthy children at same age was 10.5 times higher (OR 4.7, 95\%, CI 0.92-24.5) at 8 years of age. Oxygen treatment $>30$ days adversely affected the scores up to 18th month (OR 2.1,\%95, CI 0.44-9.8). Babies having low scores of the18th month-cognitive and motor sub-test of BSID-II had 16 times higher probability of having low WISC-R total IQ scores at 8 years. $(p<0.05) .19$ children with sepsis at 8 years had lower performance and total IQ scores $(\mathrm{p}<0.05)$.

Conclusion Prolonged oxygen therapy and having and sepsis are significant factors affecting later IQ of VLBW infants. Lower BSID-II scores at 18 th month may predict future lower total IQ scores. Longitudional follow up and early intervention is of paramount importance.

\section{PO-0400 NEUROPROTECTIVE EFFECT OF PENTOXIFYLLINE IN RAT PUPS WITH HYPOXIC-ISCHAEMIC ENCEPHALOPATHY}

${ }^{1} \mathrm{H}$ Halis, ${ }^{1} \mathrm{~T}$ Gunes, ${ }^{2} \mathrm{~N}$ Liman, ${ }^{1} \mathrm{O}$ Bastug, ${ }^{3} \mathrm{~S}$ Bitiktas, ${ }^{3} \mathrm{~B}$ Tan, ${ }^{4} \mathrm{C}$ Süer. ${ }^{1}$ Neonatal Intensive Care Unit, Erciyes University Faculty of Medicine, Kayseri, Turkey; ${ }^{2}$ Histology and Embryology, Faculty of Veterinary Medicine, Kayseri, Turkey; ${ }^{3}$ Physiology, Erciyes University School of Medicine, Kayseri, Turkey; ${ }^{4}$ Physiology, Erciyes University Faculty of Medicine, Kayseri, Turkey 